\title{
Diffusion-Weighted MRI: Distinction of Skull Base Chordoma From Chondrosarcoma
}

\author{
K.W. Yeom, R.M. Lober, B.C. Mobley, G. Harsh, H. Vogel, R. Allagio, M. Pearson, M.S.B. Edwards, and N.J. Fischbein
}

\begin{abstract}
BACKGROUND AND PURPOSE: Chordoma and chondrosarcoma of the skull base are rare tumors with overlapping presentations and anatomic imaging features but different prognoses. We hypothesized that these tumors might be distinguished by using diffusionweighted MR imaging.
\end{abstract}

\begin{abstract}
MATERIALS AND METHODS: We retrospectively reviewed 19 patients with pathologically confirmed chordoma or chondrosarcoma who underwent both conventional and diffusion-weighted MR imaging. Differences in distributions of ADC were assessed by the Kruskal-Wallis test. Associations between histopathologic diagnosis and conventional MR imaging features (T2 signal intensity, contrast enhancement, and tumor location) were assessed with the Fisher exact test.

RESULTS: Chondrosarcoma was associated with the highest mean ADC value $\left(2051 \pm 261 \times 10^{-6} \mathrm{~mm}^{2} / \mathrm{s}\right)$ and was significantly different from classic chordoma $\left(1474 \pm 117 \times 10^{-6} \mathrm{~mm}^{2} / \mathrm{s}\right)$ and poorly differentiated chordoma $\left(875 \pm 100 \times 10^{-6} \mathrm{~mm}^{2} / \mathrm{s}\right)(P<.001)$. Poorly differentiated chordoma was characterized by low T2 signal intensity $(P=.001)$, but other conventional MR imaging features of enhancement and/or lesion location did not reliably distinguish these tumor types.
\end{abstract}

CONCLUSIONS: Diffusion-weighted MR imaging may be useful in assessing clival tumors, particularly in differentiating chordoma from chondrosarcoma. A prospective study of a larger cohort will be required to determine the value of ADC in predicting histopathologic diagnosis.

ABBREVIATIONS: $\mathrm{PD}=$ poorly differentiated

C hordoma and chondrosarcoma constitute most primary bone tumors arising within the skull base, with a collective incidence of 0.03 cases per 100,000 person-years in the United States. ${ }^{1}$ Due to their common location, they often have similar clinical presentations, though recurrence and survival rates are notably worse for chordoma than for the rarer chondrosarcoma. ${ }^{2-4}$

Chordoma arises from notochordal remnants and is typically

Received June 2, 2012; accepted after revision July 26.

From the Departments of Radiology (K.W.Y., N.J.F.), Neurosurgery (R.M.L., G.H., M.S.B.E.), and Pathology (H.V.), Stanford University, Palo Alto, California; Departments of Pathology (B.C.M.) and Neurosurgery (M.P.), Vanderbilt University Medical Center, Nashville, Tennessee; and Department of Pathology (R.A.), University of Padova, Padova, Italy.

Previously presented in abstract form at: 22nd Annual Meeting of the North American Skull Base Society, February 17-19, 2012; Las Vegas, Nevada.

Please address correspondence to Kristen W. Yeom, MD, Department of Radiology, Pediatric MRI and CT, Lucile Packard Children's Hospital, Stanford University, Room 0511, 725 Welch Rd, Palo Alto, CA 94304; e-mail: kyeom@stanford.edu

三 Indicates article with supplemental on-line table.

http://dx.doi.org/10.3174/ajnr.A3333 centered on the clivus, ${ }^{5}$ whereas chondrosarcoma is mesenchymal in origin and is typically centered on the petro-occipital fissure. $^{2}$ These are not pathognomonic features, however, due to unpredictable patterns of origin, growth, and invasion. ${ }^{6}$

Microscopically, chordoma is a moderately cellular neoplasm composed of vacuolated physaliferous cells arranged singly and in cords within a myxoid stroma. ${ }^{7}$ While mitotic activity is rare in typical chordoma, poorly differentiated chordoma shows high mitotic activity and relatively high cell attenuation with a high nuclear-to-cytoplasmic ratio and prominent nucleoli. ${ }^{8-10}$ The poorly differentiated chordoma is a particularly aggressive tumor with a predilection for the pediatric population. Chondrosarcoma, histologically distinct from chordoma, is composed of atypical chondrocytes with enlarged hyperchromatic nuclei set in an abundant cartilaginous matrix. Chondrosarcoma is graded from low to high, with higher grade tumors demonstrating increased cellularity, mitotic activity, nuclear size, nuclear hyperchromasia, and aggressiveness. ${ }^{11}$

Despite distinct origins and generally distinct histopathologic 

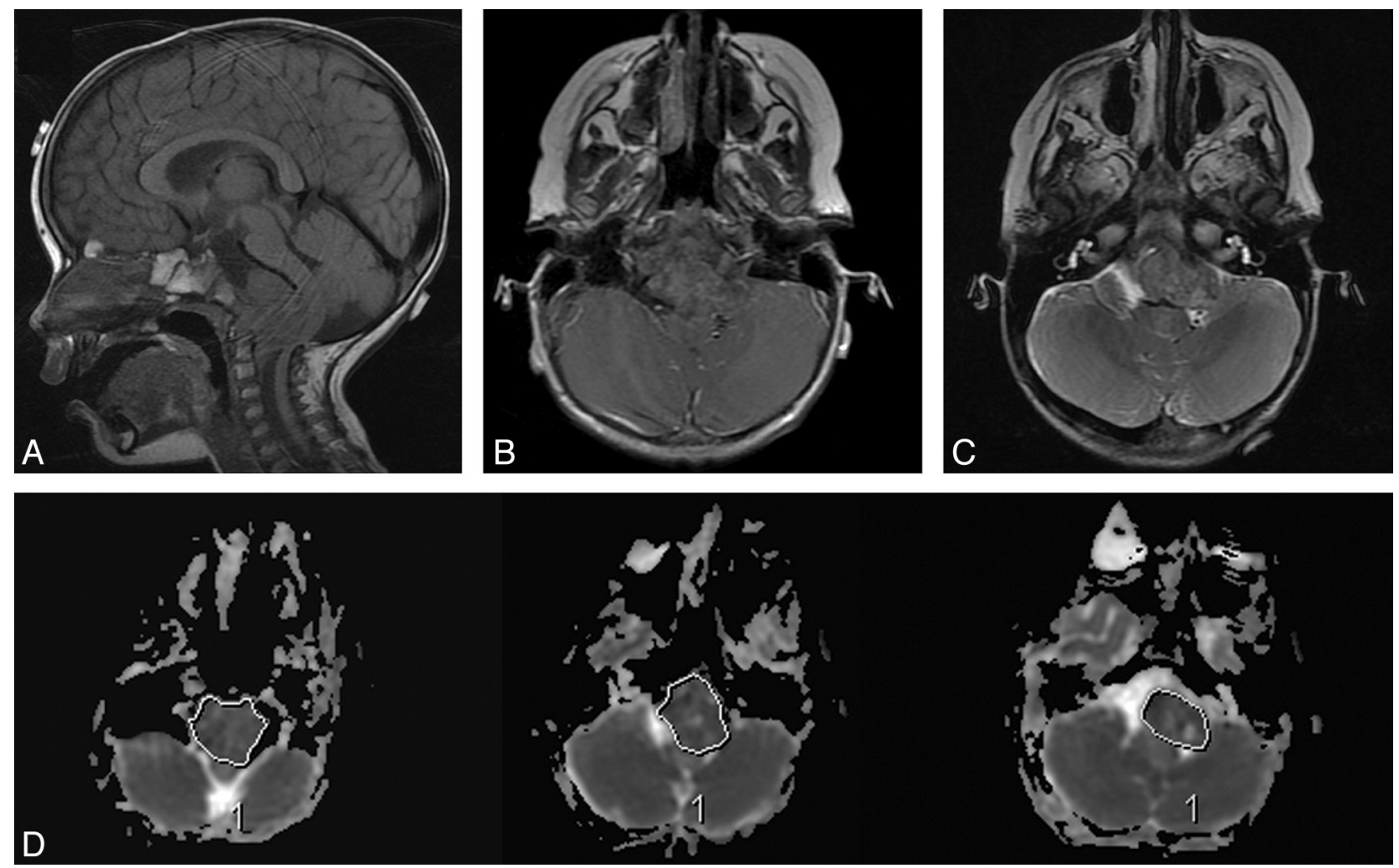

FIG 1. ADC measurement. In this 22-month-old girl, poorly differentiated chordoma is seen arising from the clivus $(A)$, which shows minimal or no enhancement $(B)$ and relatively low T2 intensity $(C)$. Regions of interest were drawn at all available axial planes of the tumor on the ADC map $(D)$ and along the entire boundary of the tumor soft tissue, avoiding areas of hemorrhage, necrosis, cyst, or calcification.

features and clinical behavior, chordoma and chondrosarcoma may be difficult to distinguish from each other on preoperative imaging. Several previous studies have found no MR imaging or CT features that definitively distinguish chordoma from chondrosarcoma. ${ }^{6,12,13}$ None of these studies, however, applied DWI, a technique used in tumor imaging to assess differences in cellular density and the nuclear-to-cytoplasmic ratio on the basis of water motion within tissue voxels. ${ }^{14-16}$ Given the discrete histopathologic features of classic chordoma, poorly differentiated chordoma, and chondrosarcoma, we hypothesized that these tumors could be distinguished by using DWI.

\section{MATERIALS AND METHODS \\ Subjects}

All patients with pathologically proved skull base chordoma or chondrosarcoma at 2 institutions, between 2004 and 2011, were retrospectively reviewed after approval by the institutional review board, and a waiver of consent was authorized. Patient privacy was protected in accordance with the Health Insurance Portabil-
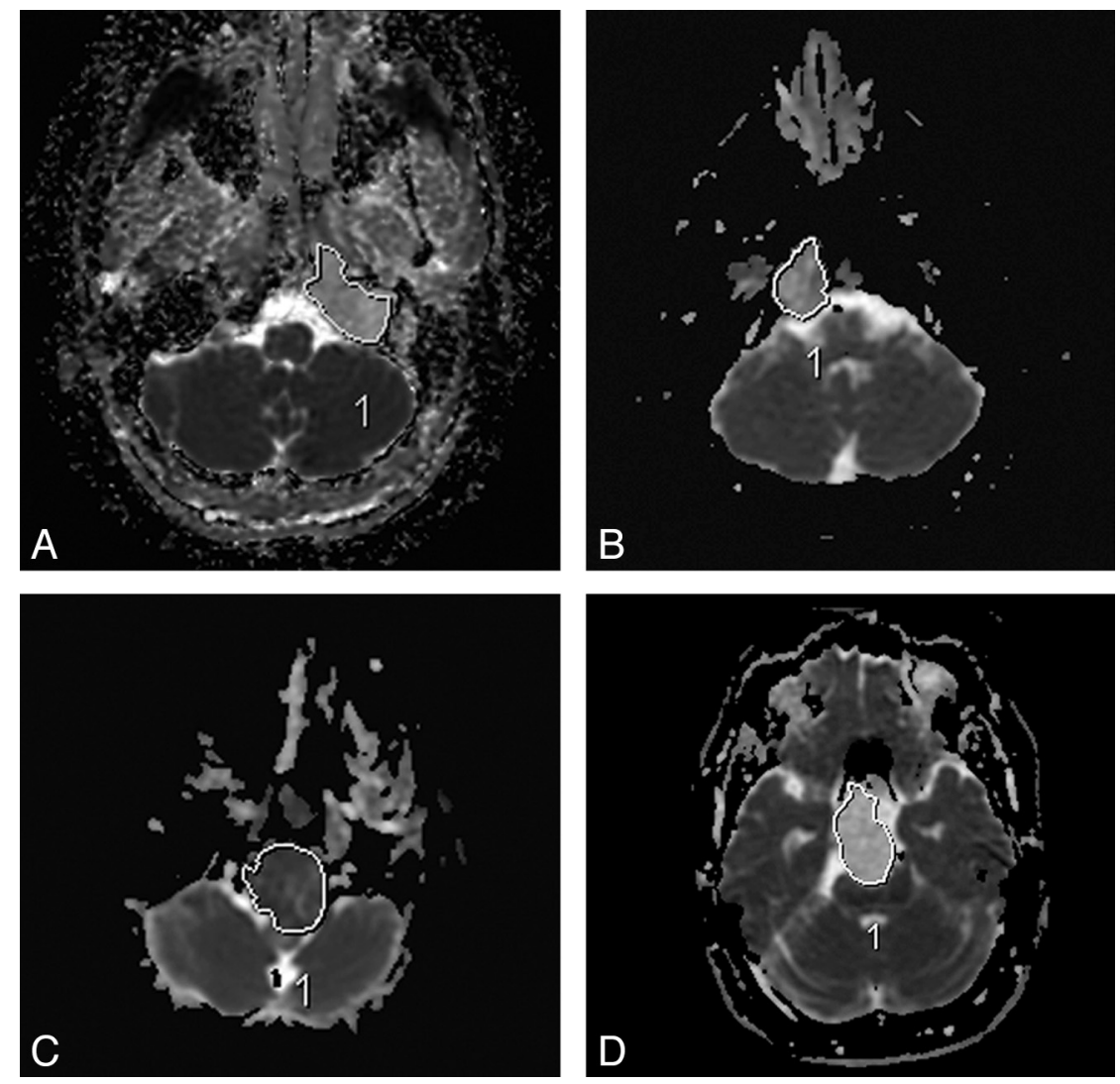

FIG 2. Sample $A D C$ measurements in various tumors. Chondrosarcoma ( $A$ and $D$ ), classic chordoma $(B)$, and poorly differentiated chordoma $(C)$. 

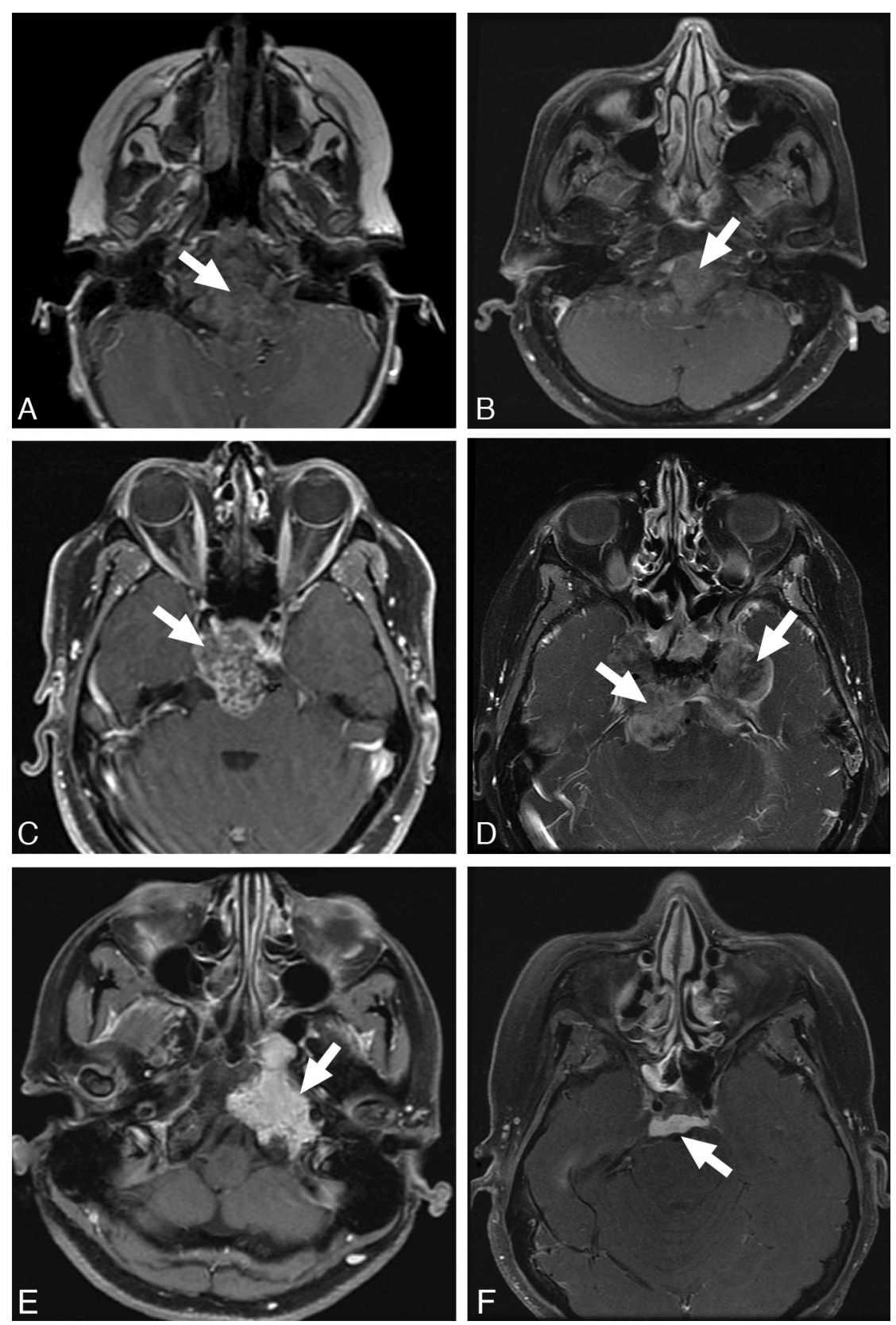

FIG 3. Enhancement patterns. Examples of enhancement patterns, mild to none (top), heterogeneous (middle), and solid (bottom) are shown in poorly differentiated chordomas ( $A$ and $D$ ), classic chordoma $(B)$, and chondrosarcoma $(C, E$, and $F)$.

ity and Accountability Act of 1996 and with our institutional ethics standards and guidelines. The study cohort was identified from a consecutive series of patients in a pathology data base that included 2 institutions and was defined by 2 inclusion criteria: the patient underwent both conventional and diffusion-weighted MR imaging preoperatively and had pathology confirmed by surgical resection.

\section{Imaging Methods}

Of our 19 patients, 16 were examined with brain MR imaging at $1.5 \mathrm{~T}$ or $3 \mathrm{~T}$ field strength (Signa, Discovery 750; GE Healthcare, Milwaukee, Wisconsin) with the following brain MR imaging parameters: T2 FSE (TR/TE, 2700-3000/100-120 ms); FLAIR (TR/ TE/TI, 9000/120/2200 ms); T1 spin-echo pre- and postgadolinium injection (TR/TE, 600-700/15-20 ms at $5-\mathrm{mm}$ section thickness with a 0 - to 1-mm skip); and DWI (TR/TE, 8300/70 $\mathrm{ms}$ at $1.5 \mathrm{~T}$ and $10,000 / 80 \mathrm{~ms}$ at $3 \mathrm{~T}, b=$ $1000 \mathrm{~s} / \mathrm{mm}^{2}$, three directions, 4 - to $5-\mathrm{mm}$ thickness, 0 skip). Three patients were examined with brain MR imaging at $1.5 \mathrm{~T}$ (Achieva; Philips Healthcare, Best, the Netherlands) with the following brain MR imaging parameters: T2 FSE (TR/TE, 5000/100 ms); FLAIR (TR/TE/TI, 10,000/ 140/2200 ms); T1 spin-echo pre- and postgadolinium injection (TR/TE, $500-$ 600/15 $\mathrm{ms}$ at 5-mm thickness, 1 skip); and DWI (TR/TE, 4000/53 ms, $b=1000$ $\mathrm{s} / \mathrm{mm}^{2}$, three directions, $5-\mathrm{mm}$ thickness, 0 skip). ADC maps were generated with commercially available software.

\section{Radiologic Evaluation}

The ADC measurements were obtained from regions of interest that encompassed the soft-tissue component of the tumor. In 2 cases, patients with large intratumoral hemorrhages were excluded because nonhemorrhagic tumor could not be defined for measurement. ROIs were drawn by a board-certified neuroradiologist with a Certificate of Added Qualification (K.W.Y.), blinded to both pathologic and clinical information. A region of interest was drawn on all axial images that included the tumor, excluding areas of cyst/cavity formation, hemorrhage/mineralization, and/or necrosis (Figs 1 and 2) identified on the anatomic imaging sequences. Mean, maximum, and minimum ADC values were obtained within this region of interest at all axial sections that included the tumor and then averaged. Region-of-interest placement was also performed in a blinded fashion by a neurosurgeon (R.M.L.) on the basis of 6 random sample cases; interobserver agreement for ADC values was analyzed by using the intraclass correlation coefficient and was found to be highly reliable (95\% confidence interval, 0.979-1.000).

Additionally, we investigated the following MR imaging features: enhancement pattern, T2 intensity relative to gray matter, and lesion location. Enhancement pattern was graded as $0=$ little or none, $1=$ heterogeneous, or $2=>90 \%$ homogeneous enhancement (Fig 3). Lesions were scored as high or low T2 intensity on the basis of homogeneous areas that were characteristic of $\geq 90 \%$ of the tumor volume (Fig 4). One case (patient 16 in the On-line Table) was excluded from the analysis with the Fisher exact test due to mixed T2 intensity, and this is noted as a heterogeneous lesion containing varying T2 intensities in the On-line 

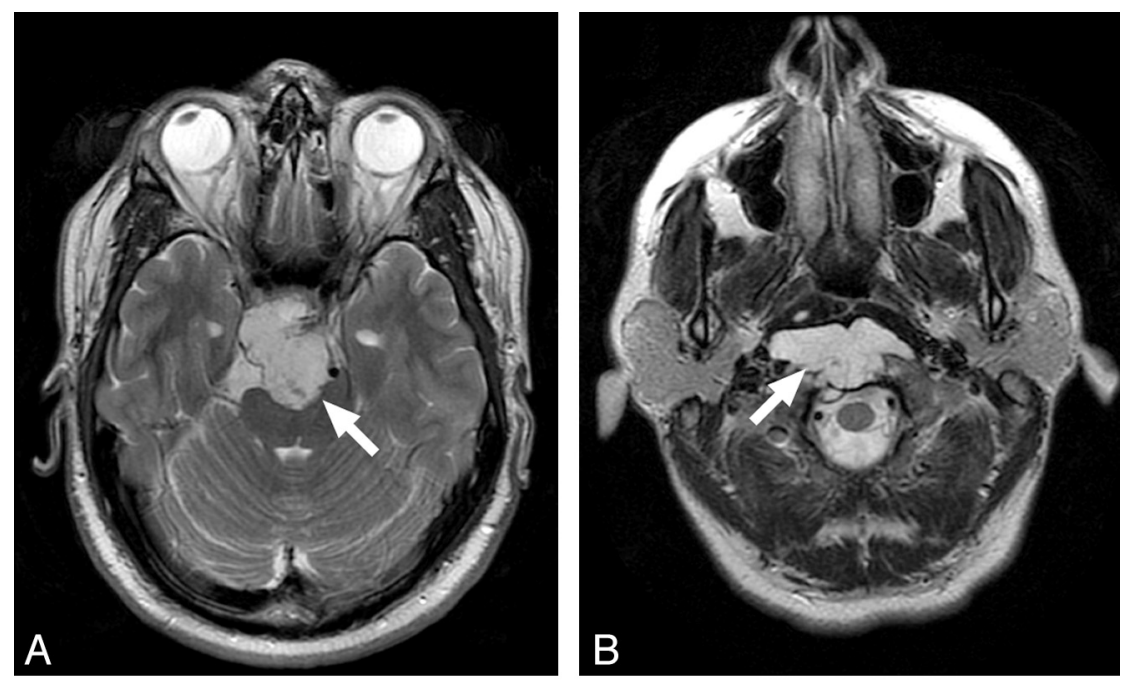

wise comparisons. Associations between histopathologic diagnosis and MR imaging features of T2-weighted signal, contrast enhancement, and tumor location were assessed with the Fisher exact test.

\section{RESULTS \\ Subjects}

Of 38 patients identified from our data bases, 21 patients had available diffusionweighted imaging. Two patients with large intratumoral hemorrhage were excluded due to difficulty visualizing nonhemorrhagic soft-tissue components of tumor. The characteristics and clinical data of the remaining 19 patients are shown in Table 1. Individual demo-
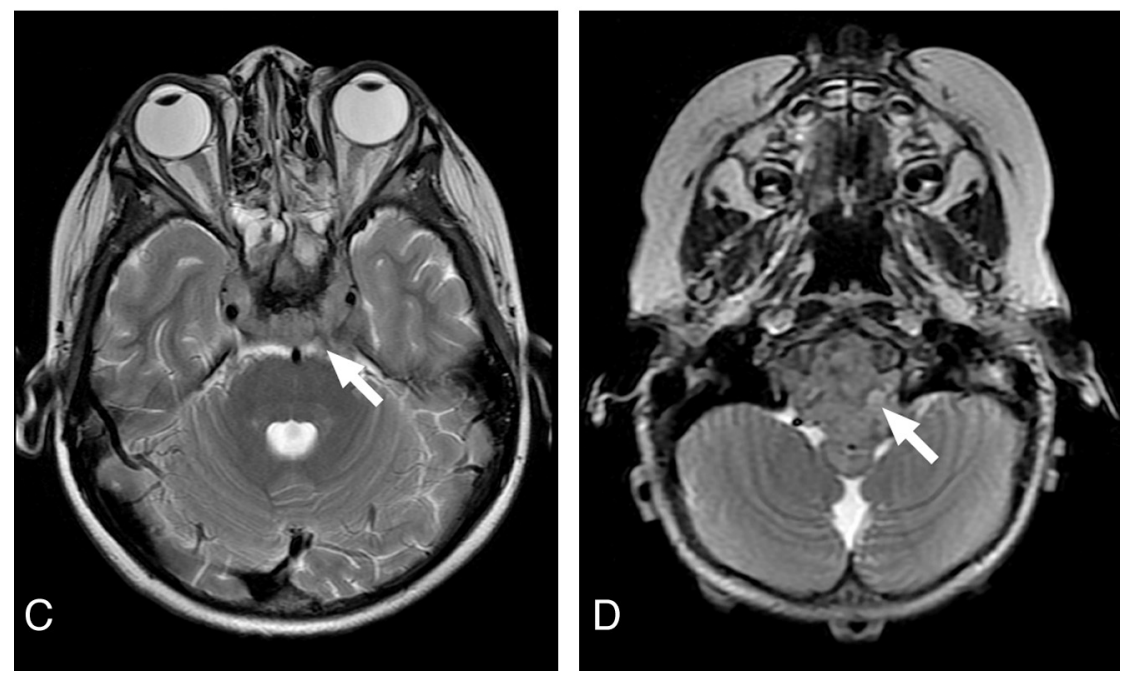
graphic and MR imaging features of each tumor are shown in the On-line Table. The median age at diagnosis was 36 (range, 2-84) years, and there were 8 males and 11 females. Diagnoses included $9(47 \%)$ chondrosarcomas, 7 (37\%) classic chordomas, and 3 (16\%) poorly differentiated chordomas. Histologic analysis included immunohistochemical studies, with chondrosarcoma demonstrating S-100 reactivity and lack of keratin staining, and chordoma demonstrating brachyury reactivity. Loss of nuclear SMARCB1/INI1 reactivity was observed in the poorly differentiated subset of chordoma. Tumor locations included 11 (58\%) clival, 7 (37\%) petroclival, and 1 (5\%) pterygoid regions. Sixteen (84\%) of the tumors were new diagnoses, and 3 (16\%) represented residual or recurrent

FIG 4. Examples of $\mathrm{T} 2$ intensity features of the clival tumors. High $\mathrm{T} 2$ intensity of chondrosarcoma $(A)$ and classic chordoma $(B)$ are easily recognized. Contrast this with the low T2 intensity of poorly differentiated chordoma $(C$ and $D)$.

Table. These MR imaging features were assessed by a board-certified neuroradiologist (K.W.Y.), again blinded to pathologic and clinical information.

\section{Pathology}

Tumor specimens were retrieved from the surgical pathology files of Stanford University Medical Center and Vanderbilt University Medical Center. Histologic diagnoses were confirmed by boardcertified neuropathologists (H.V., B.C.M.). Immunohistochemical staining for SMARCB1/INI1, brachyury, cytokeratin CKAE1/ AE3, and S-100 was performed per standard protocols by using formalin-fixed paraffin-embedded tissue. The appropriate positive and negative tissue controls were analyzed.

\section{Statistical Analysis}

All statistical analyses were performed with the Statistical Package for the Social Sciences, Version 20.0 (SPSS, Chicago, Illinois) with an a priori significance level of $\alpha=.05$. Differences in distributions of ADC and patient age for the 3 tumor types were assessed by the Kruskal-Wallis test of independent samples with multiple pair- disease. Two of the 3 patients with recurrent/residual tumors had been treated with radiation before imaging.

Patient age at diagnosis differed among the groups, with significance due to the difference between poorly differentiated chordoma and chondrosarcoma $(P=.047)$. The mean ages in years at diagnosis for chondrosarcoma, classic chordoma, and poorly differentiated chordoma were $43.3 \pm 17.3,37.7 \pm 23$, and $5.7 \pm 3.2$ years, respectively.

\section{Diffusion MR Imaging}

The distributions of averaged mean, minimum, and maximum ADC for the 3 tumor types are shown in Table 2. Chondrosarcoma had the highest ADC values, followed by classic chordoma, and PD chordoma, which had the lowest ADC values. The tumor types differed in mean, minimum, and maximum ADC according to the Kruskal-Wallis test of independent samples $(P=.001$, 0.001 , and 0.028 , respectively). For mean and minimum ADC, pair-wise comparisons identified differences between chondrosarcoma and both classic and PD chordoma, while these values were not different between the 2 chordoma types. For maximum 
Table 1: Patient characteristics and clinical data $(n=19)$

\begin{tabular}{|c|c|c|c|c|}
\hline & $\begin{array}{l}\text { Entire Cohort } \\
\qquad(n=19)\end{array}$ & $\begin{array}{l}\text { Chondrosarcoma } \\
(n=9)\end{array}$ & $\begin{array}{l}\text { Classic Chordoma } \\
(n=7)\end{array}$ & $\begin{array}{l}\text { Poorly Differentiated } \\
\text { Chordoma }(n=3)\end{array}$ \\
\hline \multicolumn{5}{|l|}{ Age at diagnosis (yr) } \\
\hline Median & 36 & 37 & 36 & 7 \\
\hline Range & $2-84$ & $28-84$ & $2-64$ & $2-8$ \\
\hline \multicolumn{5}{|l|}{ Sex } \\
\hline Male & $8(42 \%)$ & $3(33 \%)$ & $3(43 \%)$ & $2(67 \%)$ \\
\hline Female & $11(58 \%)$ & $6(67 \%)$ & $4(57 \%)$ & $1(33 \%)$ \\
\hline \multicolumn{5}{|l|}{ Tumor location } \\
\hline Clival & $11(58 \%)$ & $4(44 \%)$ & $5(71 \%)$ & $3(100 \%)$ \\
\hline Petroclival & $7(37 \%)$ & $4(44 \%)$ & $2(29 \%)$ & $0(0 \%)$ \\
\hline Pterygoid & $1(5 \%)$ & $1(11 \%)$ & $0(0 \%)$ & $0(0 \%)$ \\
\hline \multicolumn{5}{|c|}{ New or recurrent/residual } \\
\hline New & $16(84 \%)$ & $8(89 \%)$ & $5(71 \%)$ & $3(100 \%)$ \\
\hline Recurrent/residual & $3(16 \%)$ & $1(11 \%)$ & $2(29 \%)$ & $0(0 \%)$ \\
\hline
\end{tabular}

Table 2: ADC values for each tumor type $\left(10^{-6} \mathrm{~mm}^{2} / \mathrm{s}\right)$

\begin{tabular}{cccc}
\hline Tumor & $\begin{array}{c}\text { Mean ADC } \\
\text { (Median) }\end{array}$ & $\begin{array}{c}\text { Minimum ADC } \\
\text { (Median) }\end{array}$ & $\begin{array}{c}\text { Maximum ADC } \\
\text { (Median) }\end{array}$ \\
\hline Chondrosarcoma & $2051 \pm 262$ & $1488 \pm 360$ & $2503 \pm 512$ \\
$(n=9)$ & $(1977)$ & $(1352)$ & $(2392)$ \\
Classic Chordoma & $1474 \pm 117$ & $905 \pm 118$ & $2199 \pm 255$ \\
$(n=7)$ & $(1460)$ & $(860)$ & $(2217)$ \\
Poorly Differentiated & $875 \pm 100$ & $491 \pm 210$ & $1503 \pm 127$ \\
Chordoma $(n=3)$ & $(871)$ & $(469)$ & $(1557)$ \\
\hline
\end{tabular}

Table 3: MRI features for each tumor type

\begin{tabular}{cccc}
\hline \multicolumn{1}{c}{ Tumor } & $\begin{array}{c}\text { T2 } \\
\text { Hypointensity }\end{array}$ & $\begin{array}{c}>90 \% \\
\text { Enhancement }\end{array}$ & $\begin{array}{c}\text { Clival } \\
\text { Location }\end{array}$ \\
\hline $\begin{array}{c}\text { Chondrosarcoma } \\
(n=9)\end{array}$ & $0(0 \%)$ & $5(56 \%)$ & $4(44 \%)$ \\
$\begin{array}{c}\text { Classic chordoma } \\
(n=7)\end{array}$ & $0(0 \%)$ & $1(14 \%)$ & $4(57 \%)$ \\
$\begin{array}{c}(n=0) \\
\text { Poorly differentiated } \\
\text { chordoma }(n=3)\end{array}$ & $3(100 \%)$ & $0(0 \%)$ & $3(100 \%)$ \\
\hline
\end{tabular}

ADC, the $P$ value was significant because of the difference between PD chordoma and chondrosarcoma. Distributions of mean, minimum, and maximum ADC values were not different between new and residual or recurrent disease $(P=.911,0.576$, and 0.441 , respectively).

\section{Other MR imaging Features}

Additional MR imaging features are shown in Table 3. Among the tumor types, poorly differentiated chordoma was distinguished by T2 low intensity relative to gray matter $(P=.001)$. Classic chordoma and chondrosarcoma demonstrated T2 high intensity (Fig 4), except in 1 case that showed mixed intensity. The tumor types were not distinguished by contrast enhancement $(P=.167)$ or clival-versus-petroclival location $(P=.381)$. Only 1 tumor had an area of MR imaging-visible calcification or focal blood product, and this area was excluded from region-of-interest measurement.

\section{DISCUSSION}

In this study, chondrosarcoma was associated with the highest ADC values (mean ADC: $2051 \pm 261 \times 10^{-6} \mathrm{~mm}^{2} / \mathrm{s}$ ), followed by classic chordoma $\left(1474 \pm 117 \times 10^{-6} \mathrm{~mm}^{2} / \mathrm{s}\right)$ and poorly differentiated chordoma $\left(875 \pm 100 \times 10^{-6} \mathrm{~mm}^{2} / \mathrm{s}\right)$. Multiple pair- wise comparisons demonstrated significant ADC differences between chondrosarcoma and both types of chordoma but did not distinguish the 2 chordoma types. These differences likely reflect tumor biology (eg, differences in cellular density, nuclear-to-cytoplasmic ratio, and extracellular matrix, each of which has been shown to contribute to nonuniformity of ADC in other tumor types $\left.^{14-17}\right)$.

Diffusion measurements within an imaging voxel reflect the movement of both extracellular and intracellular water. ${ }^{15,17} \mathrm{Hy}-$ percellular areas may have low ADC values from reduced water motion within a crowded interstitial space. ${ }^{14}$ Similarly, tumor cells with high nuclear-to-cytoplasmic ratio and prominent nucleoli may have low ADC from reduced water motion within cells because of increased intracellular protein. ${ }^{18}$

Among these skull base tumors, the striking differences in ADC may be attributable to the extracellular matrices of these tumors. The higher ADC values of chondrosarcomas, which have varying degrees of cellularity within a cartilaginous stroma, ${ }^{2,19-21}$ likely reflect relatively free extracellular water motion. Our ADC value for chondrosarcomas is very similar to the value of $2290 \times$ $10^{-6} \mathrm{~mm}^{2} / \mathrm{s}$ reported by Hayashida et $\mathrm{al}^{22}$ for chondrosarcomas outside the skull base, which have identical histologic features. In contrast, the myxoid stroma of chordomas, through which strands or sheets of physaliferous cells are arranged, likely impedes extracellular water motion to a greater degree.

In the case of very low diffusion, the diagnosis of poorly differentiated chordoma may be considered because water motion is further reduced in this markedly more cellular tumor compared with classic chordoma and chondrosarcoma. This subtype, however, may be difficult to distinguish from other highly cellular neoplasms of the skull base, such as rhabdomyosarcoma and primitive neuroectodermal tumor, and requires a pathologic assessment with special stains such as those for brachyury and INI- $1 .{ }^{10}$

For tumors in general, variation in ADC may arise from heterogeneity in cell size and density, as well as microscopic hemorrhage or calcification. In our study, 2 hemorrhagic tumors were excluded because nonhemorrhagic tumor could not be defined for measurement, and in 1 case with MR imaging-visible focal calcification or blood product, region-of-interest placement and imaging section selection were conducted carefully to exclude this area from analysis. 
Our study corroborates previous reports that both classic chordoma and chondrosarcoma show high intensity on T2weighted images. ${ }^{6,12,13}$ Our cohort included 3 cases of poorly differentiated chordoma, and these demonstrated $\mathrm{T} 2$ low intensity relative to gray matter, a finding that, to our knowledge, has not been previously reported. We found no pattern of contrast enhancement that distinguished among tumor types, similar to previous reports. ${ }^{6}$

We recognize that a larger sample size might demonstrate overlapping diffusion characteristics among these tumors, just as there may be overlapping histologic features on microscopic examination, but large sample sizes of these rare tumors are difficult to accrue. The chondroid variant of chordoma, for example, may show stromal features that mimic the hyaline cartilage seen in chondrosarcoma, ${ }^{2}$ while a subset of chondrosarcoma can show a myxoid matrix characteristic of chordoma. Both tumors may have areas of calcification and even ossification. ${ }^{2}$ Because of the close relationship between histology and tissue water properties, ADC may fail as an absolute predictor of diagnosis in such cases of histopathologic crossover and variability but can still potentially provide worthwhile information about the presumptive diagnosis of a skull base lesion. A further limitation may be our use of patient data following radiation therapy, which may increase the variance of $\mathrm{ADC}$ values and limit the applicability for preoperative diagnosis. However, despite higher ADC among irradiated chordomas, the values were still lower than those of any chondrosarcoma.

Diffusion properties of skull base tumors previously have not been compared for the purpose of preoperative diagnosis, except in 1 recent study in which ADC was the basis for a general distinction between benign and malignant skull lesions that correlated with cell density. ${ }^{23}$ Our results suggest that diffusion-weighted imaging and calculation of ADC values may aid in distinguishing chordoma from chondrosarcoma.

\section{CONCLUSIONS}

Diffusion MR imaging may be useful in assessing clival tumors, particularly in differentiating chordoma from chondrosarcoma and suggesting the rare poorly differentiated chordoma. Prospective study of a larger cohort will be required to determine the value of $\mathrm{ADC}$ in predicting the histopathologic diagnosis.

\section{ACKNOWLEDGMENTS}

The authors would like to recognize the excellent technical support and impeccable service of staff members in our Departments of Neurosurgery and Radiology.

Disclosures: Nancy Fischbein—UNRELATED: Board Membership: AJNR Senior Editor.

\section{REFERENCES}

1. Central Brain Tumor Registry of the United States. Statistical Report: Primary Brain Tumors in the United States, 1997-2001. Hinsdale, Illinois: Central Brain Tumor Registry of the United States
2. Heffelfinger MJ, Dahlin DC, MacCarty CS, et al. Chordomas and cartilaginous tumors of the skull base. Cancer 1973;32:410-20

3. Almefty K, Pravdenkova S, Colli BO, et al. Chordoma and chondrosarcoma: similar, but quite different, skull base tumors. Cancer 2007;110:2456-67

4. Cho YH, Kim JH, Khang SK, et al. Chordomas and chondrosarcomas of the skull base: comparative analysis of clinical results in $\mathbf{3 0}$ patients. Neurosurg Rev 2008;31:35-43

5. Burrow JF, Stewart MJ. Original papers: malignant spheno-occipital chordoma. J Neurol Psychopathol 1923;15:205-17

6. Pamir MN, Ozduman K. Analysis of radiological features relative to histopathology in $\mathbf{4 2}$ skull-base chordomas and chondrosarcomas. Eur J Radiol 2006;58:461-70

7. Mirra JM, Nelson SD, Della Rocca C, et al. Chordoma. In: Fletcher CDM, Unni KK, Mertens F, eds. World Health Organization Classification of Tumours: Pathology and Genetics of Tumours of Soft Tissue and Bone. Lyon, France: IARC Press; 2002:315-17

8. Hoch BL, Nielsen GP, Liebsch NJ, et al. Base of skull chordomas in children and adolescents: a clinicopathologic study of 73 cases. Am J Surg Pathol 2006;30:811-18

9. Coffin CM, Swanson PE, Wick MR, et al. Chordoma in childhood and adolescence: a clinicopathologic analysis of 12 cases. Arch Pathol Lab Med 1993;117:927-33

10. Mobley BC, McKenney JK, Bangs CD, et al. Loss of SMARCB1/INI1 expression in poorly differentiated chordomas. Acta Neuropathol 2010;120:745-53

11. Bertoni F, Bacchini P, Hogendoorn PC. Chondrosarcoma. In: Fletcher CDM, Unni KK, Mertens F, eds. World Health Organization Classification of Tumours: Pathology and Genetics of Tumours of Soft Tissue and Bone. Lyon, France: IARC Press; 2002:247-51

12. Oot RF, Melville GE, New PF, et al. The role of MR and CT in evaluating clival chordomas and chondrosarcomas. AJR Am J Roentgenol 1988;151:567-75

13. Bourgouin PM, Tampieri D, Robitaille Y, et al. Low-grade myxoid chondrosarcoma of the base of the skull: CT, MR, and histopathology. J Comput Assist Tomogr 1992;16:268-73

14. Sugahara T, Korogi Y, Kochi M, et al. Usefulness of diffusionweighted MRI with echo-planar technique in evaluation of cellularity in gliomas. J Mag Res Imaging 1999;9:53-60

15. Guo AC, Cummings TJ, Dash RC, et al. Lymphomas and high-grade astrocytomas: Comparison of water diffusibility and histologic characteristics. Radiology 2002;224:177-83

16. Rumboldt Z, Camacho DL, Lake D, et al. Apparent diffusion coefficients for differentiation of cerebellar tumors in children. AJNR Am J Neuroradiol 2006;27:1362-69

17. Le Bihan D, Breton E, Lallemand D, et al. MR imaging of intravoxel incoherent motions: Application to diffusion and perfusion in neurologic disorders. Radiology 1986;161:401-07

18. Filippi CG, Edgar MA, Ulug AM, et al. Appearance of meningiomas on diffusion-weighted images: correlating diffusion constants with histopathologic findings. AJNR Am J Neuroradiol 2001;22:65-72

19. Lichtenstein L, Jaffe HL. Chondrosarcoma of bone. Am J Pathol 1943;19:553-89

20. Dahlin DC, MacCarty CS. Chordoma. Cancer 1952;5:1170-78

21. Evans HL, Ayala AG, Romsdahl MM. Prognostic factors in chondrosarcoma of bone: a clinicopathologic analysis with emphasis on histologic grading. Cancer 1977;40:818-31

22. Hayashida Y, Hirai T, Yakushiji T, et al. Evaluation of diffusionweighted imaging for the differential diagnosis of poorly contrastenhanced and T2-prolonged bone masses: initial experience. J Magn Reson Imaging 2006;23:377-82

23. Ginat DT, Mangla R, Yeaney G, et al. Diffusion-weighted imaging for differentiating benign from malignant skull lesions and correlation with cell density. AJR Am J Roentgenol 2012:198:W597-601 